\title{
Penile Melanoma
}

National Cancer Institute

\section{Source}

National Cancer Institute. Penile Melanoma. NCI Thesaurus. Code C162547.

A melanoma that arises from the penis. It may be cutaneous or mucosal. The most common site of involvement is glans penis. 